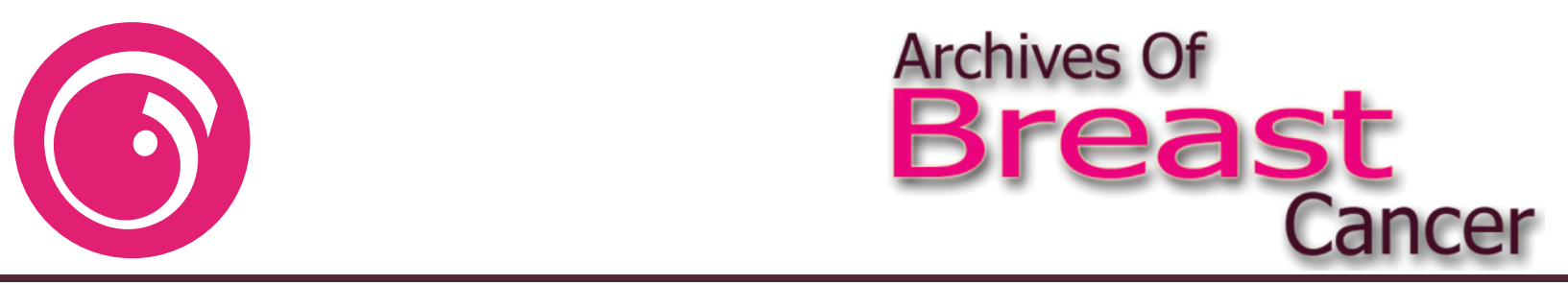

DOI: $10.32768 / a b c .20218121-28$

\title{
Patient Throughput Times for Supplemental Breast Cancer Screening Exams
}

\author{
Michael J. Plaza*a, Denzel Cole ${ }^{b}$, Marcos A. Sanchez-Gonzalez ${ }^{b}$, Christopher J. Starr ${ }^{c}$ \\ ${ }^{a}$ Department of Radiology, Diagnostic Center for Women, Miami, Florida, USA \\ ${ }^{b}$ Department of Radiology, Larkin Community Hospital, Miami, Florida, USA \\ ${ }^{\circ}$ Department of Radiology, Kaiser Permanente Downey Medical Center, Miami, Florida, USA
}

\section{ARTICLE INFO \\ Received: \\ 22 October 2010 \\ Revised: \\ 01 December 2020 \\ Accepted: \\ 04 December 2020}

Key words:

Breast MRI,

patient flow,

patient throughput,

breast cancer screening,

breast ultrasound

\section{ABSTRACT}

Background: To optimize screening abbreviated breast MRI (ABMR) operations, patient throughput times of $\mathrm{ABMR}$ were compared to breast ultrasound (US) and full protocol breast MRI (FPMR).

Methods: Patient throughput times (mean \pm standard error) and its subcomponents were analyzed for 95 ABMRs, 90 breast US exams, and 50 FPMRs. Total patient throughput was measured from registration time to the time of the last acquired image. Actual exam time was time difference between the first and last acquired images and pre-examination time was the calculated difference between throughput and actual exam times.

Results: ABMR total patient throughput time was shorter than FPMR (55.7 \pm 1.7 vs. $63.1 \pm 2.0 \mathrm{~min}$; difference, $7.4 \mathrm{~min}, 13 \%$; $<<0.001$ ), but longer than breast US (39.1 $\pm 1.3 \mathrm{~min}$; difference, $16.6 \mathrm{~min}, 30 \%$; $\mathrm{p}<0.001)$. ABMR had shorter actual scan times than FPMR ( $13.4 \pm 0.14$ vs. $18.6 \pm 0.25 \mathrm{~min} ; \mathrm{p}<0.001)$, but longer than US $(9.6 \pm 0.46$ minutes; $\mathrm{p}<0.001)$. There was no difference in the preexamination times between ABMR and FPMR (42.3 \pm 1.7 vs. $44.6 \pm 1.9$ min; $\mathrm{p}=$ $0.357)$; pre-examination times were longer for both MR exam types compared to US $(29.5 \pm 1.3$ minutes; $\mathrm{p}<0.001)$.

Conclusion: ABMR patient throughput times are faster than FPMR, but these gains are limited as they have no impact on pre-examination activities which comprise the lengthiest components of the patient flow process. US patient flow currently remains faster than ABMR; however, comparable ABMR times could be achieved by further omitting certain sequences and optimizing pre-examination processes.

\section{Introduction}

Patients with heterogeneously dense or extremely dense breast tissue composition are at an increased risk for breast cancer and require supplemental breast cancer screening due to the limited sensitivity of mammography in this population. ${ }^{1}$ Since approximately $43 \%$ of women have dense breast tissue, this

Address for correspondence:

Michael J. Plaza, MD (MJP)

Diagnostic Center for Women

7500 SW 87th Ave, Suite 100, Miami, FL, 33173

Tel: +1 $3057405100,+17047795604$

Fax: +1 3054245445

Email: michael.j.plaza@gmail.com represents a large number of patients for which a mammographically occult breast cancer may be detected. $^{2}$

There are many converging lines of evidence to show that breast MRI is superior to ultrasound (US) in cancer detection and in reducing interval cancer rate in patients with dense breasts who may not meet the criteria for additional MRI screening by lifetime risk. ${ }^{3}$ The value of MRI is further made apparent by the fact that additional MRI detected cancers are more aggressive; and thus, earlier detection of this cancer subset is tantamount to better survival rates and to allow for management with less morbid systemic medical therapies and surgical management. 
Although breast MRI has been shown to be superior to breast US in cancer detection, most practices that utilize supplemental screening primarily use breast US given its availability, accessibility, and affordability. ${ }^{6}$ However, screening breast US can have high inter-operator variability leading to higher number of false positives and additional downstream costs including unnecessary follow-up and biopsies.

Full protocol breast MRI (FPMR), as it stands, faces many challenges in terms of cost, access, and availability to be utilized mainstream for screening average-risk women with dense breasts. Given these limitations, the scope of FPMR use is limited to patients with greater than $20 \%$ lifetime risk and even in this population breast MRI remains highly underutilized. ${ }^{8,9}$ Nonetheless, studies have also shown that abbreviated breast MRI (ABMR) demonstrates sensitivity and specificity in cancer detection comparable to FPMR and with improved patient throughput. ${ }^{10,11}$ Improved patient flow in radiology translates to reduced costs and greater patient access. ${ }^{12}$ Thus, utilization of ABMR in highrisk patients and those with dense breast tissues have the potential to increase cancer detection in a large number of patients that may not have otherwise been readily detected by screening mammogram or US.,11

However, to date, it is unknown to what degree ABMR improves patient flow compared to FPMR in an outpatient setting, and if gains in operational efficiency are enough such that it would be feasible to employ it in lieu of screening breast US in routine clinical practice. In this study, we examine the operations of three supplemental breast cancer exam types, technologist-performed hand-held whole breast ultrasound, ABMR, and FPMR. The purpose is to optimize screening ABMR operations via a "lean" methodology and through process maps analyses as well as compare ABMR patient throughput times to US and FPMR.

\section{Methods}

This Health Insurance Portability and Accountability Act compliant retrospective observational study was performed as a quality improvement initiative and received IRB exemption. The need to obtain informed consent was waived. We retrospectively reviewed all supplemental breast cancer screening examinations in women with normal screening mammograms from November 2019 through January 2020. This included technologist performed hand-held breast ultrasound $(\mathrm{n}=90)$, abbreviated screening breast MRI $(\mathrm{n}=95)$, and full protocol breast MRI $(\mathrm{n}=50)$. We included patients who had a return visit only for the purpose of performing the supplemental breast cancer screening examination. We excluded symptomatic patients (palpable abnormality or nipple discharge), patients with newly diagnosed breast cancer, patients with history of mastectomy, patients with supplemental exams performed the same day as the screening mammogram, and if any additional diagnostic imaging exam (i.e. DEXA, thyroid ultrasound, pelvic ultrasound, etc.) was performed the same day.

\section{Facility}

The facility is a free standing women's diagnostic imaging center and is part of an over 500-physician multispecialty group. We are a hybrid private practice/teaching facility and American College of Radiology accredited Breast Imaging Center for Excellence. The center performs over 30000 mammograms, 17000 breast ultrasounds, and over 1700 breast MRIs annually.

\section{Breast Ultrasound}

Our institutional policy is to recommend supplemental technologist-performed hand-held screening whole breast ultrasound on all women with dense breasts. Our facility has 13 breast ultrasound technologists ranging in experience from 2- 20 years; 12 of 13 are breast specialty certified by American Registry for Diagnostic Medical Sonography. We have six rooms available for performing screening breast ultrasound; five utilize a Samsung RS80A (Samsung Healthcare, San Jose, CA) and one utilizes an Epiq 5W (Philips Healthcare; Andover, MA). All technologists are required to undergo a rigorous supervised training for at least 3 weeks with a more experienced technologist prior to scanning alone. Our ultrasound protocol requires taking at least one static image of 2:00, 4:00, 6:00, 8:00, 10:00, 12:00, subareaolar, and the axilla for each breast. All cysts are documented with gray-scale and power Doppler imaging. All solid masses are documented in orthogonal planes, with and without measurement calipers, and with power Doppler imaging.

\section{Breast MRI}

All breast MRIs were performed using a single 1.5T Siemens Magnetom Espree scanner (Siemens Medical Solutions USA, Malvern, PA) with a 16channel bilateral Sentinelle breast coil (Invivo, Gaineville, FL). ABMRs are performed on all highrisk with women with normal prior MRI using the protocol described in a previous publication. ${ }^{13}$ FPMRs are performed for all baseline screening examinations in high-risk patients. We have two MRI technologists, both with over 10 years experience performing contrast-enhanced breast MRI. Expected scan time was obtained by summing the time of acquisition for each imaging series under each protocol. The FPMR expected scan time was 13.1 minutes and consists of the following sequences: localizer images, unenhanced non-fat-suppressed T1-weighted imaging, unenhanced fat-suppressed short inversion time inversion recovery (STIR) 


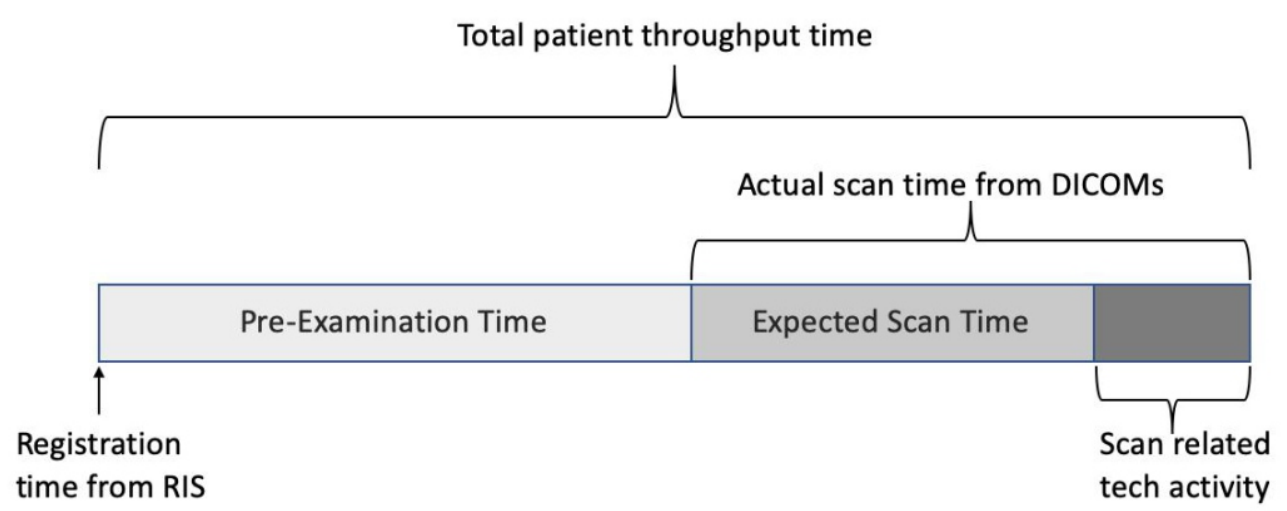

Figure 1. Time elements measured or calculated in the study. Expected scan time is only for the MR examinations.

RIS = radiology information system

imaging, unenhanced fat-suppressed gradient-echo T1-weighted imaging followed by two early phase dynamic contrast-enhanced fat-suppressed gradientecho sequences and one 6-minute postcontrast late phase sequence. The ABMR expected scan time was 9.5 minutes and consists of the following sequences: localizer images, unenhanced fat-suppressed STIR imaging, unenhanced fat-suppressed gradient-echo T1-weighted imaging followed by two continuously scanned early phase dynamic contrast-enhanced fatsuppressed gradient-echo sequences.

\section{Patient Throughput Times and Data Analysis}

Figure 1 displays the different elements recorded in measuring or calculating patient throughput times. Initial registration time representing the time of arrival was recorded from Intergy (Greenway Health, Tampa, FL), the radiology information system (RIS). We used Infinitt (Infinitt North America, Phillipsburg, $\mathrm{NJ}$ ), our picture archiving and communication systems (PACS) to record the DICOM times stamps for the first and last acquired images.

Total patient throughput time was defined as the time from registration to the time of the final acquired image. Actual scan time was the difference in time between the first and last acquired images. Pre-examination time was the difference in total patient throughput time and actual scan time. Scan related technologist activity was the difference in actual scan time and expected scan time.

We recorded patient variables that could potentially influence the study activity times, including age, breast volume, and whether or not they have breast implants. Breast volume was summed together for each breast and estimated by using the craniocaudal mammographic projection measuring the width $\mathrm{W}$ (distance from medial-to-lateral breast surfaces along the posterior edge), the posterior-toanterior height $\mathrm{H}$ (perpendicular distance from posterior edge to the nipple), and the compression thickness $\mathrm{C}$ using the follow equation 14 :

$$
\text { Volume }=0.785 \times \mathrm{H} x \mathrm{~W} \times \mathrm{C}
$$

A one-way analysis of variance (ANOVA) test was used compare mean age and mean breast volume between the three exam types. Proportions of patients with and without breast implants were compared between the three exam types using a Fisher's exact test with $3 \times 2$ contingency table. For each exam type, a Pearson's correlation test was performed to determine if there was an association between age, breast volume, or implant status that may influence any of the activity times (i.e. preexamination time, actual scan time, patient throughput time.) Mean activity times and standard error were calculated for each exam type and compared controlling for any differences in patient variables using a hierarchical multiple regression analysis. A priori $\mathrm{p}$ value of less than 0.05 was considered statistically significant for all calculations. Analyses were performed using statistical software SPSS version 22.0 (IBM; Chicago, IL).

\section{"Lean" Initiative}

After completing the data collection phase, a "lean" approach to improving operations was initiated by engaging supervisors and staff from the front-desk, ultrasound, and MRI departments in a dialogue and qualitative interview. ${ }^{15} \mathrm{~A}$ single researcher served as both interviewer and note taker. The steps involved as a patient moves through the imaging center from the time of registration to the time of the final acquired image were discussed in great detail with staff members. Questions ranged from being broad and open-ended to focused and specific in order to gain insights into inefficiencies, improve understanding of differences unique to each imaging modality, and highlight opportunities for improvement of workflow and performance. The interview guide is displayed in Table 1.

\section{Results}

Patient Characteristics

All patient characteristics are summarized in Table 2. A total of 90 female patients underwent 
Table 1. List of questions for qualitative interview of front-desk staff, ultrasound technologists, and MRI technologists.

\begin{tabular}{|c|c|c|}
\hline Staff & General questions & Deep-dive questions \\
\hline Registration & $\begin{array}{l}\text {-What is the process when a patient arrives at } \\
\text { registration? } \\
\text { - Do you have any suggestions for improving } \\
\text { efficiency? }\end{array}$ & $\begin{array}{l}\text { - Do patients usually have these forms filled out before } \\
\text { arriving? } \\
\text {-What forms do US (or MRI) patients fill out at } \\
\text { registration? } \\
\text { - How is the patient transferred to the US (MRI) } \\
\text { department? }\end{array}$ \\
\hline Ultrasound & $\begin{array}{l}\text {-What tasks do you need to complete before you } \\
\text { pick up a patient from the waiting room? } \\
\text {-What are the steps that occur when you take the } \\
\text { patient to the exam room? } \\
\text {-Do you have any suggestions for improving } \\
\text { efficiency? }\end{array}$ & $\begin{array}{l}\text {-What are sources for delay prior to getting the patient to the } \\
\text { exam room? } \\
\text {-What are the most common reasons for delays during the } \\
\text { ultrasound examination? }\end{array}$ \\
\hline MRI & $\begin{array}{l}\text {-What tasks do you need to complete before you } \\
\text { pick up a patient from registration? } \\
\text {-What tasks do you perform before positioning } \\
\text { the patient in the magnet? } \\
\text {-What steps occur after patient is positioned in the } \\
\text { magnet? } \\
\text {-Do you have any suggestions for improving } \\
\text { efficiency? }\end{array}$ & $\begin{array}{l}\text {-How are you made aware of the patient arrival? } \\
\text {-What forms and consents do you review with the patient? } \\
\text {-How long does it typically take to get IV access? Who do } \\
\text { you typically call for assistance with difficult veins and how } \\
\text { long does it take that person to arrive? } \\
\text {-What are the most common questions patient have? } \\
\text {-What sequences do you usually have to repeat? }\end{array}$ \\
\hline
\end{tabular}

supplemental screening breast US with mean age $47.4 \pm 0.8$ (mean \pm standard error) years. Mean breast volume was $1847 \pm 150 \mathrm{cc}$ and $24 \%$ (22 of 90) had breast implants. A total of 95 female patients underwent supplemental screening ABMR with a mean age of $50.9 \pm 0.78$ years. Within this group, mean breast volume was $1779 \pm 92 \mathrm{cc}$ and $16 \%(15$ of 95) had breast implants. The FPMR group included 50 female patients with a mean age of 50.5 \pm 1.5 years. Mean breast volume was $1838 \pm 73 \mathrm{cc}$ and $18 \%$ ( 9 of 50 ) had breast implants.

The ultrasound group was found to be statistically younger than the ABMR or FPMR groups $(p=0.015)$. There was no significant difference in ages between the ABMR and FPMR groups. There was no significant difference in the breast volumes $(\mathrm{p}=0.723)$ or proportion of patients with/without implants $(\mathrm{p}=0.257)$ among the different exam types.

Pearson correlation coefficients were calculated and demonstrated no significant association between age, breast volume, or implant status for any of the study activity times for US, ABMR, and FPMR exams; respectively.

\section{Patient Throughput Times}

All activity time results are summarized in Table 3. The total patient throughput times for US, ABMR, and FPMR were $39.1 \pm 1.3$ minutes (mean \pm standard error), $55.7 \pm 1.7$ minutes, and $63.1 \pm 2.0$ minutes; respectively. ABMR total patient throughput time was significantly shorter than FPMR (difference, 7.4 minutes, $13 \% ; \mathrm{p}<0.001$ ), but significantly longer than breast US (difference, 16.6 minutes, 30\%; $\mathrm{p}<0.001)$.

Table 2. Summary of patient demographics for each exam type, including age, breast volume, and implant status.

\begin{tabular}{lcccc}
\hline Demographic & US $(\mathrm{n}=90)$ & ABMR $(\mathrm{n}=95)$ & FPMR $(\mathrm{n}=50)$ & P value \\
\hline Mean Age (years) & 47.4 & 50.9 & 50.5 & 0.015 \\
Mean Breast Volume (cc) & 1847 & 1779 & 1838 & 0.723 \\
Patients with Implants (\%) & 24 & 16 & 18 & 0.257 \\
\hline
\end{tabular}

$U S=$ ultrasound, $A B M R=$ abbreviated breast MRI, FPMR $=$ full protocol breast MRI.

Table 3. Data table of mean patient throughput times and derived values in minutes for each exam type.

\begin{tabular}{lccccc}
\hline Exam type & $\begin{array}{c}\text { Patient Throughput } \\
\text { Time }\end{array}$ & $\begin{array}{c}\text { Pre-Examination } \\
\text { Time }\end{array}$ & $\begin{array}{c}\text { Actual Scan } \\
\text { Time }\end{array}$ & $\begin{array}{c}\text { Expected Scan } \\
\text { Time }\end{array}$ & $\begin{array}{c}\text { Scan related } \\
\text { Technologist Activity }\end{array}$ \\
\hline US & 39.1 & 29.5 & 9.6 & N/A & N/A \\
ABMR & 55.7 & 42.3 & 13.4 & 9.5 & 3.9 \\
FPMR & 63.1 & 44.6 & 18.6 & 13.1 & 5.5 \\
\hline
\end{tabular}

$U S=$ ultrasound, $A B M R=$ abbreviated breast $M R I, F P M R=$ full protocol breast $M R I, N / A=$ not applicable 
Actual scan times yielded similar results. ABMR actual scan time was significantly longer than breast US $(13.4 \pm 0.14$ minutes versus $9.6 \pm 0.46$ minutes; difference, 3.8 minutes, $28 \%$; $<0.001$ ), but significantly shorter than FPMR $(13.4 \pm 0.14$ minutes versus $18.6 \pm 0.25$ minutes; difference, 5.2 minutes, $39 \% ; \mathrm{p}<0.001)$. Scan related technologist activity times were statistically shorter for ABMR versus FPMR (3.9 versus 5.5 minutes; difference, 1.6 minutes; $\mathrm{p}<0.001)$.

Pre-examination times were longer for the MRI examinations $(\mathrm{ABMR}=42.3 \pm 1.7$ minutes, $\mathrm{FPMR}=$ $44.6 \pm 1.9$ minutes $)$ compared to breast US $(29.5 \pm 1.3$ minutes; difference 12.8 minutes, $30 \%$; $\mathrm{p}<0.001$ ). However, there was no significant difference in preexamination times between ABMR and FPMR $(\mathrm{p}=$ $0.357)$.

Hierarchical logistic regression analysis confirmed that differences in study activity times were not impacted by any variations in age, breast volume, or implant status between the different exam types.

\section{“Lean” Process Qualitative Interview}

Staff interviews performed as part of the "lean" initiative allowed for the development of process maps for each exam type (Figure 2). This then facilitated the identification of sources of variations in the study activity times. It was clear that a patient coming in for supplemental screening breast MRI had several additional steps unique to the modality. At the registration desk, this included MRI specific forms which although patients are asked to fill out before arriving, most do not. US patients are taken by registration staff to the diagnostic waiting area and are already changed into a robe when the technologist calls upon the patient; which is in stark contrast to MRI patients who are taken from registration to the MRI suite by the technologist and then the MRI technologist waits for the patient to change into a robe. Intravenous (IV) access, gadolinium consent, and patient questions/concerns regarding gadolinium deposition are additional steps unique to the pre-examination times for both

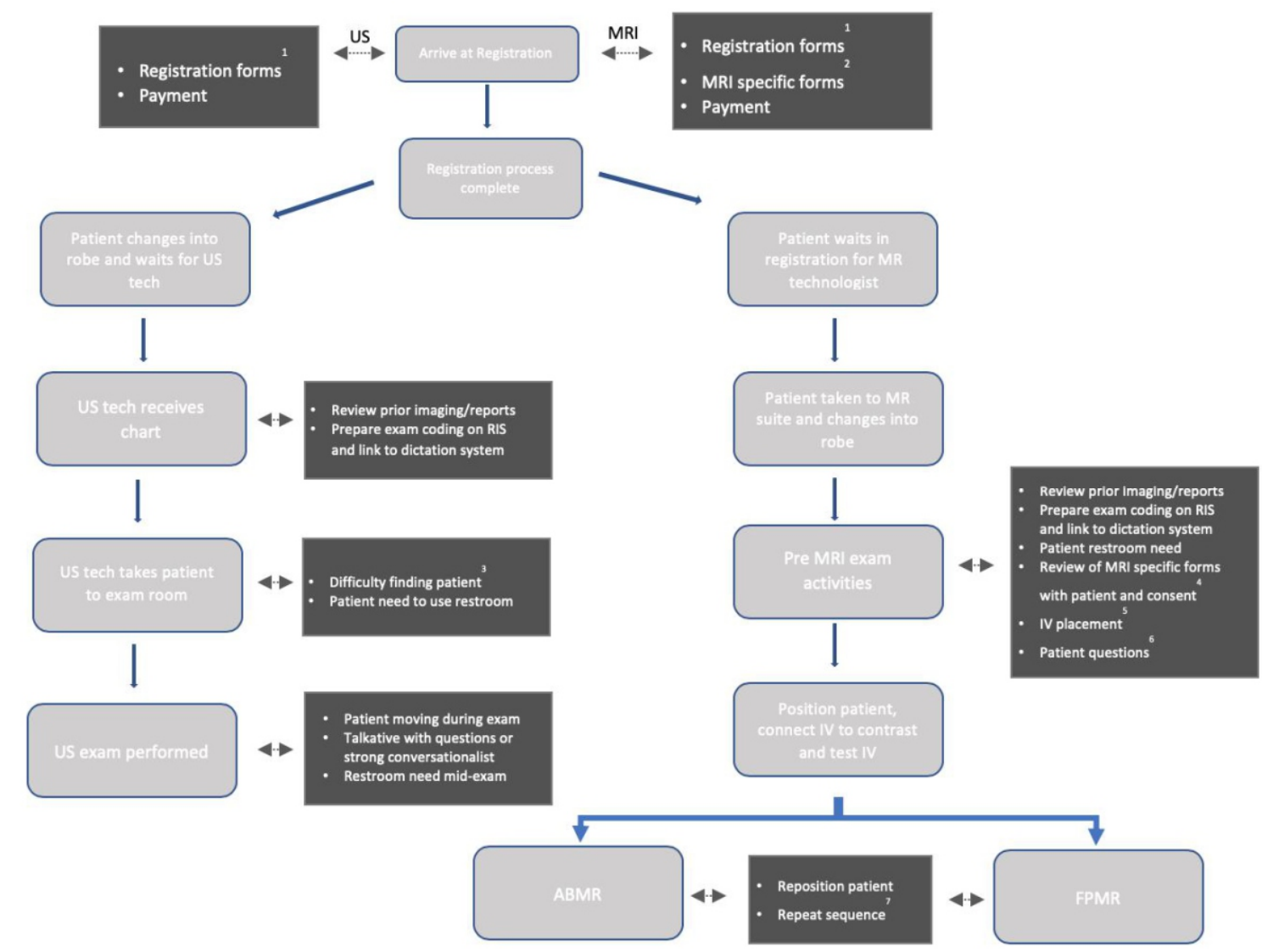

Figure 2. Ultrasound and MRI process maps beginning from registration time to final acquired image. Dark rectangles include elements identified at that time point which may be sources for variations in the activity times. US = ultrasound, RIS = radiology information system, IV = intravenous access, $\mathrm{ABMR}=$ abbreviated breast MRI, FPMR $=$ full protocol MRI.

${ }^{1}$ Forms include notice of privacy, financial policy, and medical record release.

${ }^{2}$ MRI forms include clinical history and MR safety questionnaire, consent to MRI and use of gadolinium contrast, insurance authorization disclaimer specific to MRI.

${ }^{3}$ Patients go to use restroom, leave to make phone calls, go missing in the center, or fail to answer when called by the technologist due to being distracted.

${ }^{4}$ MRI forms given in registration are reviewed by the technologist with the patient. Forms often incomplete or filled out incorrectly.

${ }^{5}$ IV access performed by MRI technologist sometimes requires multiple sticks or nurse is needed to be called for additional assistance.

${ }^{6}$ Majority of questions pertaining to MRI/magnet safety and gadolinium safety/deposition.

$7 \mathrm{Need}$ to repeat sequences for motion or for suboptimal fat saturation in implants/large breasts. 
ABMRs and FPMRs. Difficulty obtaining IV access can result in significant delays if the nurse needs to be called for assistance and is not readily available. There were no difference in the pre-examination processes described for patients having an ABMR or FPMR. MRI technologists subjectively described that the need to repeat sequences most commonly occurred with large almost entirely fatty breasts and implants due to inhomogeneous fat suppression.

\section{Discussion}

Implementing ABMR into our outpatient clinical practice for high risk patients has resulted in faster overall patient throughput times and shorter magnet time compared to FPMR. As one may expect, there was no difference in MRI pre-examination times which is in keeping with the recorded steps of the process map (Figure 2) and with Borthakur and colleagues' prior analysis of ABMRs in an academic hospital setting. ${ }^{16}$ Our study demonstrated ABMR to have a $13 \%$ faster overall patient flow rate compared to FPMR. This gain in operational efficiency translates to increased capacity, improved patient access, reduced unit cost, and increased potential for revenue growth. ${ }^{12,17}$ ABMR demonstrated a $39 \%$ reduction in magnet time compared to FPMR which is postulated to result in better patient care through improved compliance and less image motion artifact especially for patients who suffer from claustrophobia. ${ }^{10}$ The operational gains from ABMR at our institution may be even greater at other sites as our FPMR is already under 15 minutes, while other literature describes typical standard full protocol breast MR to be in the range of 20-30 minutes. ${ }^{10,16}$ In addition, although not formally recorded in our analysis, an ABMR inherently results in acquiring fewer images reducing image storage costs, allowing for faster interpretation, and less likelihood of repetitive injury of the interpreting radiologist. ${ }^{10,18}$

Patient throughput times for ultrasound were 30\% faster compared to ABMR with an average difference of 16.6 minutes. This difference could be further reduced to 10.2 minutes by eliminating the STIR and second post-contrast sequence which would be expected to have negligible impact on screening metrics. Most published literature on ABMR describe protocols without a $\mathrm{T} 2$ weighted sequence or second post contrast sequence yielding similar accuracy to FPMR. ${ }^{11}$ The added value of T2-weighted imaging remains controversial, but there is data suggesting its value is only minimal as it may only change the final assessment in as little as 3\% of exams. ${ }^{11,19}$ Lean interview and process map analysis identified preexamination operational inefficiencies of ABMRs. These include MR technologists waiting on patients to change into a robe, delays in IV access, and delays due heightened patient concerns and questions regarding gadolinium safety. Implementation of changes to address these forms of muda and mura would only have to improve processes by approximately 10 minutes on average to achieve ABMR throughput times comparable to US screening. ${ }^{15}$

A time-driven activity-based costing analysis is beyond the scope of this manuscript, but it is intuitive that ABMR is less costly than FPMR as the unit cost parameters are no different, while the unit time required is clearly less as demonstrated in our study. ${ }^{20}$ Screening breast MRI has been shown to be cost-effective in high-risk populations and implementation of ABMR in this population would only add to its value. ${ }^{21,}{ }^{22}$ As mentioned earlier implementing ABMR also has the side-effect of improving capacity and patient accessibility, which is an important consideration given that screening breast MRI remains significantly underutilized by high-risk population despite its proven benefits. ${ }^{9}$

The question remains if the time gains achievable through ABMR are enough to offset the lower cost of US if implemented in an average risk population with dense breasts. This would require a costeffectiveness analysis as the benefit of detecting 716 additional mammographically occult cancers by MRI versus 2-4 additional US-detected cancers per 1000 women screened would need to offset the higher cost of MRI. ${ }^{3,23-25}$ Without a CPT code for an ABMR exam, reimbursement and widespread adaptation in an average risk population remains a challenge. The mean cost of a breast US in the United States is $\$ 134$ versus $\$ 1,197$ for a standard full protocol breast MRI. ${ }^{26}$ Our results imply that given the resources and time required to perform ABMR as well as the known greater value provided to the patient in terms of greater sensitivity and fewer false positives compared to US, it is reasonable to charge at price point greater than US, but less than a FPMR. ${ }^{27}$ Institutional dependent out-of-pocket prices are in the range of $\$ 250-\$ 500$ which is supported by our results and further corroborated by using other self-pay advanced imaging screening tests such as lung cancer CT screening and cardiac CT scoring as precedents. ${ }^{3,11}$

We had hypothesized that age would reflect how agile a patient is and therefore how quickly they move through the center thus affecting activity times. We also expected that a larger breast volume would require longer US scan times as more breast tissue has to be covered and that implants could results in longer MRI times due to difficulties in positioning or achieving homogeneous fat saturation. However, none of these patient factors impacted activity times in our cohort, although the US group was found to be slightly younger than the MR groups. Perhaps sample size was too small to detect statistically significant differences or there are other demographics worthy of exploration that may be more impactful on throughput times such as Karnofsky or ECOG performance status or self- 
reported claustrophobia. ${ }^{28,29}$

There were several limitations to our study. This was performed at a community practice in a single outpatient free-standing imaging center where over $90 \%$ of the volume is breast imaging. These exact times may not be generalizable to other practice types, but the principle that ABMR is more operationally efficient than FPMR and that throughput times comparable to screening breast US are potentially attainable is universal. Another limitation is that pre-examination times were not separated to record specific time spent during registration prior to being seen by the technologist. Subjectively, interviews of the front-desk staff unanimously agreed that there was no difference in the time take it takes to register an US screening or breast MRI screening patient. Another limitation is that we did not account for variability of patient arriving early or late to their appointment. However, all patients are uniformly asked to arrive 30 minutes prior to their appointment time and we assume that this variability is ingrained into the acquired data and is reported in the standard error of the times.

Furthermore, we do not discuss implemented improvements in the identified inefficiencies of ABMR patient throughput. This was due to the time at which the data was collected which was subsequently followed by the COVID-19 pandemic. Operations and patient volumes have drastically changed in our institution just as it has in others since the pandemic, such that a comparison of patient throughput times pre- and post-pandemic would be fraught with bias. ${ }^{30,31}$ Nevertheless, these results provide insight comparing patient throughput times of different supplemental breast cancer screening exams and in view of the pandemic come at a critical time when healthcare resources are limited and the value of radiology exams are under careful scrutiny. This is an opportune time for greater widespread implementation ABMR in at least the high-risk population to reduce backlogs due to the pandemic and increase accessibility. All stakeholders stand to benefit, but policy makers must recognize that a billable code for ABMR is prerequisite to routine clinical use.

In conclusion, ABMR demonstrates clear operational gains compared to FPMR in the outpatient setting. Despite inherent processes unique to MRI exams, ABMR patient throughput times comparable to US screening are likely attainable by further selecting out unnecessary imaging sequences and identifying and eliminating operational waste at one's institution. A larger multicenter study would be an essential next step to ensure the reproducibility of our results across multiple practice types.

\section{Acknowledgements}

We would like to thank Dianelys Paez, Llileydy
Yanes, Niurka Alvarado, Milena Rodriguez, Nancy Moreno for their assistance in data collection.

\section{Conflict of Interest}

None of the authors have any conflicts of interest.

\section{References}

1. Kerlikowske K, Zhu W, Tosteson AN, Sprague BL, Tice JA, et al. Identifying women with dense breasts at high risk for interval cancer: a cohort study. Annals of internal medicine. 2015; 162(10):673-81.

2. Sprague BL, Gangnon RE, Burt V, TrenthamDietz A, Hampton JM, et al. Prevalence of mammographically dense breasts in the United States. JNCI: Journal of the National Cancer Institute. 2014;106-110.

3. Berg WA, Rafferty EA, Friedewald SM, Hruska CB, Rahbar H. Screening Algorithms in Dense Breasts: AJR Expert Panel Narrative Review. American Journal of Roentgenology. 2020:1-20.

4. Berg WA, Zhang Z, Lehrer D, Jong RA, Pisano $E D$, et al. Detection of breast cancer with addition of annual screening ultrasound or a single screening MRI to mammography in women with elevated breast cancer risk. JAMA. 2012;307(13):1394-404.

5. Sung JS, Stamler S, Brooks J, Kaplan J, Huang T, et al. Breast cancers detected at screening MR imaging and mammography in patients at high risk: method of detection reflects tumor histopathologic results. Radiology. 2016;280(3):716-22.

6. Choudhery S, Patel BK, Johnson M, Geske J, Fazzio RT, et al. Trends of Supplemental Screening in Women With Dense Breasts. J Am Coll Radiol. 2020;17(8):990-8.

7. Berg WA, Vourtsis A. Screening breast ultrasound using handheld or automated technique in women with dense breasts. Journal of Breast Imaging. 2019;1(4):283-96.

8. Lee CS, Monticciolo DL, Moy L. Screening Guidelines Update for Average-Risk and HighRisk Women. Am J Roentgenol. 2020;214(2):31623.

9. Miles R, Wan F, Onega TL, Lenderink-Carpenter A, O'Meara ES, et al. Underutilization of supplemental magnetic resonance imaging screening among patients at high breast cancer risk. Journal of Women's Health. 2018;27(6): 748-54.

10. Harvey SC, Di Carlo PA, Lee B, Obadina E, Sippo D, et al. An Abbreviated Protocol for High-Risk Screening Breast MRI Saves Time and Resources. JAm Coll Radiol. 2016;13(4):374-80.

11. Heacock L, Reig B, Lewin AA, Toth HK, Moy L, et al. Abbreviated breast MRI: road to clinical implementation. Journal of Breast Imaging. 2020; 2(3):201-14.

12. O'Brien JJ, Stormann J, Roche K, Cabral- 
Goncalves I, Monks A, et al. Optimizing MRI logistics: focused process improvements can increase throughput in an academic radiology department. American Journal of Roentgenology. 2017;208(2):W38-44.

13. Plaza MJ, Perea E, Sanchez-Gonzalez MA. Abbreviated Screening Breast MRI in Women at Higher-than-Average Risk for Breast Cancer with Prior Normal Full Protocol MRI. Journal of Breast Imaging. 2020;2(4):343-51.

14. Kalbhen CL, McGill JJ, Fendley PM, Corrigan $\mathrm{KW}$, Angelats J. Mammographic determination of breast volume: comparing different methods. Am J Roentgenol. 1999;173(6):1643-9.

15. Kruskal JB, Reedy A, Pascal L, Rosen MP, Boiselle PM. Quality initiatives: lean approach to improving performance and efficiency in a radiology department. Radiographics. 2012; 32(2):573-87.

16. Borthakur A, Weinstein SP, Schnall MD, Conant EF. Comparison of study activity times for "full" versus "fast MRI" for breast cancer screening. Journal of the American College of Radiology. 2019;16(8):1046-51.

17.Patient Throughput: A Critical Strategy for Success." The Chartis Group.2007. [Available from: http://www.chartis.com/resources/files/whitepapers/pre -2013/chartis group patient-throughput-criticalstrategy-for-success.pdf

18. Thompson AC, Prill MJK, Biswal S, Rebner M, Rebner RE, et al. Factors associated with repetitive strain, and strategies to reduce injury among breast-imaging radiologists. Journal of the American College of Radiology. 2014;11(11): 1074-9.

19. Dialani V, Tseng I, Slanetz PJ, Fein-Zachary V, Phillips J, et al. Potential role of abbreviated MRI for breast cancer screening in an academic medical center. Breast J. 2019;25(4):604-11.

20. Shankar PR, Hayatghaibi SE, Anzai Y. TimeDriven Activity-Based Costing in Radiology: An Overview. J Am Coll Radiol. 2020;17(1 Pt B):125-30.

21. Geuzinge HA, Obdeijn I-M, Rutgers EJ, Saadatmand S, Mann RM, et al. Costeffectiveness of Breast Cancer Screening With Magnetic Resonance Imaging for Women at Familial Risk. JAMA oncology. 2020;6(9): 1381-9.

22. Taneja C, Edelsberg J, Weycker D, Guo A, Oster $\mathrm{G}$, et al. Cost effectiveness of breast cancer screening with contrast-enhanced MRI in highrisk women. JAm Coll Radiol. 2009;6(3):171-9.

23. Bakker MF, de Lange SV, Pijnappel RM, Mann RM, Peeters PHM, et al. Supplemental MRI Screening for Women with Extremely Dense Breast Tissue. N Engl J Med. 2019;381(22): 2091-102.

24.Comstock CE, Gatsonis C, Newstead GM,
Snyder BS, Gareen IF, et al. Comparison of abbreviated breast MRI vs digital breast tomosynthesis for breast cancer detection among women with dense breasts undergoing screening. Jama. 2020;323(8):746-56.

25. Kuhl CK, Strobel K, Bieling H, Leutner C, Schild $\mathrm{HH}$, et al. Supplemental Breast MR Imaging Screening of Women with Average Risk of Breast Cancer. Radiology. 2017;283(2):361-70.

26. Vlahiotis A, Griffin B, Stavros AT, Margolis J. Analysis of utilization patterns and associated costs of the breast imaging and diagnostic procedures after screening mammography. Clinicoecon Outcomes Res. 2018;10:157-67.

27.Marshall H, Pham R, Sieck L, Plecha D. Implementing abbreviated MRI screening into a breast imaging practice. American Journal of Roentgenology. 2019;213(1):234-7.

28. Chadderdon AL, Carns DR, Pudalov LR, McKernan LC, Honce JM. Underlying Mechanisms of Psychological Interventions in Magnetic Resonance Imaging and ImageGuided Radiology Procedures. Top Magn Reson Imaging. 2020;29(3):157-63.

29. Chow R, Bruera E, Temel JS, Krishnan M, Im J, et al. Inter-rater reliability in performance status assessment among healthcare professionals: an updated systematic review and meta-analysis. Supportive Care in Cancer. 2020:;28(5):2071-8.

30. Collado-Mesa F, Kaplan SS, Yepes MM, Thurber MJ, Behjatnia B, et al. Impact of COVID-19 on breast imaging case volumes in South Florida: A multicenter study. Breast J. 2020.

31. Prabhakar AM, Glover Mt, Schaefer PW, Brink JA. Academic Radiology Departmental Operational Strategy Related to the Coronavirus Disease 2019 (COVID-19) Pandemic. J Am Coll Radiol. 2020;17(6):730-3. 